\title{
Off-line elimination of carbohydrates for amino acid analysis of samples with high carbohydrate content by ion-exchange chromatography
}

\author{
Yongsheng Ding ${ }^{\mathrm{a}}$, Hong $\mathrm{Yu}^{\mathrm{a}, \mathrm{b}}$, Shifen $\mathrm{Mou}^{\mathrm{a}, *}$ \\ ${ }^{\mathrm{a}}$ Research Center for Eco-Environmental Sciences, Chinese Academy Science, P.O. Box 2871, Beijing 100085, China \\ ${ }^{\mathrm{b}}$ Department of Chemistry, Harbin Normal University, Harbin 150080, China
}

\begin{abstract}
This paper introduces a new off-line sample preparation that eliminates carbohydrates from amino acid samples containing a high carbohydrate content before analysis by anion-exchange chromatography and integrated pulsed amperometric detection. First, the sample is introduced into a cation-exchange column in the hydrogen form. Carbohydrates are removed completely using $0.02 \%$ formic acid as a transfer fluid, while only amino acids are retained. Amino acids are then extracted from the cation-exchange resin by $10 \mathrm{ml}$ of $1 \mathrm{M}$ ammonia. The ammonia collected is evaporated to dryness and the residue redissolved in water containing $20 \mathrm{mg} / 1 \mathrm{NaN}_{3}$ for injection. All amino acids are recovered following the carbohydrate removal step. The average recovery is $97.2 \%$. The relative standard deviation for seven replicates is less than $5.2 \%$. The usefulness of the method is illustrated with chromatograms of ratafia samples obtained before and after the off-line removal of carbohydrates
\end{abstract}

(C) 2003 Elsevier Science B.V. All rights reserved.

Keywords: Carbohydrates; Amino acids

\section{Introduction}

It is important to determine amino acids in various matrices such as biological tissues/fluids, foods and environmental samples. At present, amino acids in samples of protein hydrolysate are analyzed without derivatization by anion-exchange chromatography (AEC) with integrated pulsed amperometric detection (IPAD) [1,2]. This method has advantages over pre- and post-column derivatization beyond the reduction in sample preparation and instrumentation complexity. The simplicity of the direct detection

\footnotetext{
*Corresponding author. Tel.: + 86-10-6284-9182; fax: +86-106284-9239.

E-mail address: shifenm@mail.rcees.ac.cn (S. Mou).
}

technique makes it applicable to the simultaneous determination of amino acids and sugars in samples such as cell culture media [3] and liquid condiment [4]. Ding et al. [5] developed a gradient to separate amino acids and sugars (glucose, fructose and sucrose) at different retention times, avoiding interference between sugars and amino acids. They applied this gradient to the determination of amino acids and sugars in green tea. In addition, bi-modal integrated amperometric detection with different detection potentials was introduced to distinguish between coeluting amino acids (no hydroxyl group) and carbohydrates [3]. Simultaneous separation and detection is advantageous for samples containing approximately equimolar quantities of amino acids and sugars, but samples containing uneven levels of amino acids 
and sugars are obviously challenging for the AECIPAD method. Not all methods are suitable for samples containing a large amount of carbohydrates with trace amino acids because the peaks of the amino acids merge into the large peaks of the carbohydrates. It is critical to eliminate carbohydrates prior to amino acid analysis in these samples (e.g. vegetable or fruit juices). Jandik et al. [6] introduced an automated in-line method to remove carbohydrates before amino acid analysis of carrot juice by AEC-IPAD. In their work, they used a cation-exchange resin to capture the amino acids under conditions where carbohydrates would not be retained, but two inert six-port valves and an extra sampling pump are required, which brings difficulties with respect to instrument configuration. In addition, they only verified that the carbohydrates were completely removed at a 100 -fold molar excess relative to amino acids. It is possible that weakly retained amino acids are lost in the presence of a large amount of carbohydrates beyond a 100-fold molar excess relative to amino acids.

In this work, our objective was to establish a simple and manipulable off-line sample preparation technique due to a shortage of instrumentation for in-line sample preparation. We optimized the conditions, allowing the widest possible range of carbohydrates to be completely removed by the cationexchange resin. The method was applied to amino acid analysis in a ratafia sample, which has a large amount of carbohydrates (the molar ratio of carbohydrates to amino acids can be up to 100 000:1).

\section{Experimental}

\subsection{Chemicals}

Amino acid standards were purchased from KangDa Chemicals (Shanghai, China). Sugar standards were purchased from Sigma-Aldrich (St. Louis, MO, USA). Sodium hydroxide (low carbonate), ammonia solution (analytical grade, 25\%), hydrochloric acid (analytical grade, 36\%) and formic acid (analytical grade, 88\%) were purchased from Beijing Chemical (Beijing, China). Anhydrous sodium acetate $(>99.9 \%)$ was obtained from Dionex (Sunnyvale, CA, USA). Poly(styrene-divinylbenzene) (PS-
DVB) $003 \times 7$ [size $0.3-0.4 \mathrm{~mm}$, capacity $1.8 \mathrm{mmol} /$ $\mathrm{ml}$ (wet)] resin was purchased from Nankai University Chemical Plant (Tianjin, China). Standard mixtures were prepared by diluting the stock solutions to the desired concentration with the diluent containing $20 \mathrm{mg} / \mathrm{l}$ sodium azide. All water used in this work was purified using a laboratory water purification system (Barnstead, IA, USA) and was $18 \mathrm{M} \Omega \mathrm{cm}$ in specific resistance or better. The ratafia sample was purchased from a local supermarket.

\section{2. $A E C-I P A D$}

We used a BioLC system (Dionex) consisting of a GS50 gradient pump, an AS50 autosampler with a thermal compartment and an ED50 electrochemical detector. The chromatography of amino acids was performed on an AminoPac PA10 analytical column $(250 \times 2 \mathrm{~mm}$, Dionex) at a flow-rate of $0.25 \mathrm{ml} / \mathrm{min}$ using the mobile phase gradient shown in Table 1 . The ED50 electrochemical detector uses a thin-layertype amperometric cell containing a gold electrode, a $\mathrm{pH}$ reference electrode and a titanium counter electrode. The detection waveform is presented in Table 2. PeakNet 6.30 chromatography software (Dionex) was used for system control and data analysis. The temperature of the AS50 thermal compartment was controlled at $35{ }^{\circ} \mathrm{C}$. The sample injection volume was $25 \mu l$.

Table 1

Gradient conditions

\begin{tabular}{cccc}
\hline $\begin{array}{l}\text { Time } \\
(\mathrm{min})\end{array}$ & $\begin{array}{l}\mathrm{NaOH} \\
(\mathrm{m} M)\end{array}$ & $\begin{array}{l}\mathrm{NaAc} \\
(\mathrm{m} M)\end{array}$ & Curve $^{\mathrm{a}}$ \\
\hline 0.00 & 40 & & \\
2.00 & 40 & & 8 \\
12.0 & 80 & 400 & 8 \\
16.0 & 80 & 400 & 5 \\
24.0 & 60 & & 5 \\
40.0 & 60 & & 5 \\
40.1 & 200 & & \\
42.1 & 200 & & \\
42.2 & 40 & & \\
65.0 & 40 & & \\
\hline
\end{tabular}

${ }^{\mathrm{a}}$ The shapes of the gradient curves are defined in the GS50 Gradient Pump Operator's Manual, pp. 37-38 (Dionex document No. 031612, revision 2). Curve 5 is linear and curve 8 is one of the four available concave curves (6-9) with a $20 \%$ change $\sim 60 \%$ of a time segment and a $70 \%$ change $\sim 90 \%$ of the same programmed time segment. 
Table 2

Detection waveform

\begin{tabular}{lcl}
\hline $\begin{array}{l}\text { Time } \\
(\mathrm{s})\end{array}$ & $\begin{array}{l}\text { Potential }^{\mathrm{a}}(\mathrm{V}) \\
\text { vs. pH }\end{array}$ & $\begin{array}{l}\text { Integration } \\
\text { (begin/end) }\end{array}$ \\
\hline 0.00 & 0.13 & \\
0.04 & 0.13 & Begin \\
0.05 & 0.33 & \\
0.21 & 0.33 & \\
0.22 & 0.60 & End \\
0.46 & 0.60 & \\
0.47 & 0.33 & \\
0.56 & 0.33 & \\
0.57 & -1.67 & \\
0.58 & -1.67 & \\
0.59 & 0.93 & \\
0.60 & 0.13 & \\
\hline
\end{tabular}

${ }^{\text {a }}$ Potentials applied to the Au working electrode and referenced vs. a glass $/ \mathrm{Ag} / \mathrm{AgCl}$ combination electrode.

\subsection{Off-line sample preparation}

One milliliter of PS-DVB 003x7 cation-exchange resin was packed with deionized water into a glass tube $(15 \times 0.9 \mathrm{~cm}$ I.D. $)$. Before the samples were applied to the column, the resin was treated with $4 \mathrm{ml}$ of $1 M \mathrm{HCl}$ (at least two bed volumes) then washed with deionized water until neutral. $0.25 \mathrm{ml}$ of the sample was passed through the resin at a flowrate of $0.1 \mathrm{ml} / \mathrm{min}$. Carbohydrates and interfering compounds were washed out by $30 \mathrm{ml}$ of $0.02 \%$ formic acid at a flow-rate of $1 \mathrm{ml} / \mathrm{min}$. Adjusting the flow-rate to $0.5 \mathrm{ml} / \mathrm{min}$, amino acids were extracted by $10 \mathrm{ml}$ of $1 \mathrm{M}$ ammonia solution. The ammonia solution was collected in a centrifugal tube and evaporated to dryness under a stream of nitrogen in a water bath $\left(90{ }^{\circ} \mathrm{C}\right)$. The residue was redissolved in a water solution containing $20 \mathrm{mg} / 1 \mathrm{NaN}_{3}$ for injection. The PS-DVB $003 \times 7$ resin was regenerated with $4 \mathrm{ml}$ of $1 \mathrm{M} \mathrm{HCl}$ followed by water until neutral before being reused.

\section{Result and discussion}

Depending on the $\mathrm{pH}$, amino acids can exist in one of three forms: cationic, zwitterionic or anionic. This property makes it possible to entrap amino acids as cations on the cation exchanger under acidic conditions and remove them as anions on the cation exchanger under alkaline conditions. Carbohydrates can also complex with a cation exchanger loaded with different cations, such as calcium and silver ions, by ligand exchange [7], but they are not retained on the cation-exchange resin in the hydrogen form. In this work, several sample purification conditions were optimized experimentally in order to achieve high and reproducible recoveries of amino acids. Losses of amino acids $(0.25 \mathrm{ml}, 50 \mu M$ level $)$ during sample processing were determined by a comparison with their peak areas without sample processing. Chromatograms of amino acid and sugar standards $(5 \mu M)$ are shown in Fig. 1.

\subsection{Hydrogen ionization of the cation exchanger}

PS-DVB $003 \times 7$ resin was used to eliminate carbohydrates from samples for amino acid analysis. The degree of hydrogen ionization of the resin is critical for amino acids to be retained on the cationexchange resin. Incomplete hydrogen ionization can cause a loss of amino acids, especially glutamine and asparagine. The volume of $1 \mathrm{M} \mathrm{HCl}$ used to produce hydrogen ionization of the resin should be at least two times the volume of the resin.

\subsection{Carbohydrate removal and amino acid retention}

Water is a suitable transfer fluid for removing carbohydrates [6], but the flow-rate and the volume of water have a large effect on the weakly retained amino acids, such as glutamic acid and aspartic acid. If the flow-rate is up to $1 \mathrm{ml} / \mathrm{min}$, the two acidic amino acids will be washed out entirely by $20 \mathrm{ml}$ of water. In contrast, if the flow-rate of water is lower than $0.2 \mathrm{ml} / \mathrm{min}$, the losses of glutamic acid and aspartic acid are less than about $20 \%$. At a low flow-rate, a large volume of water can also result in losses of the weakly retained amino acids. The amino acids are retained on the cation exchanger in cationic form, and are retained strongly on the resin under acidic conditions. Therefore, an attempt to reduce the loss of acidic amino acids by adjusting the $\mathrm{pH}$ of the water was carried out. The results obtained show that the losses of weakly retained amino acids were clearly reduced with an acidic transfer fluid, even at a large flow-rate $(5 \mathrm{ml} / \mathrm{min})$ with a large 


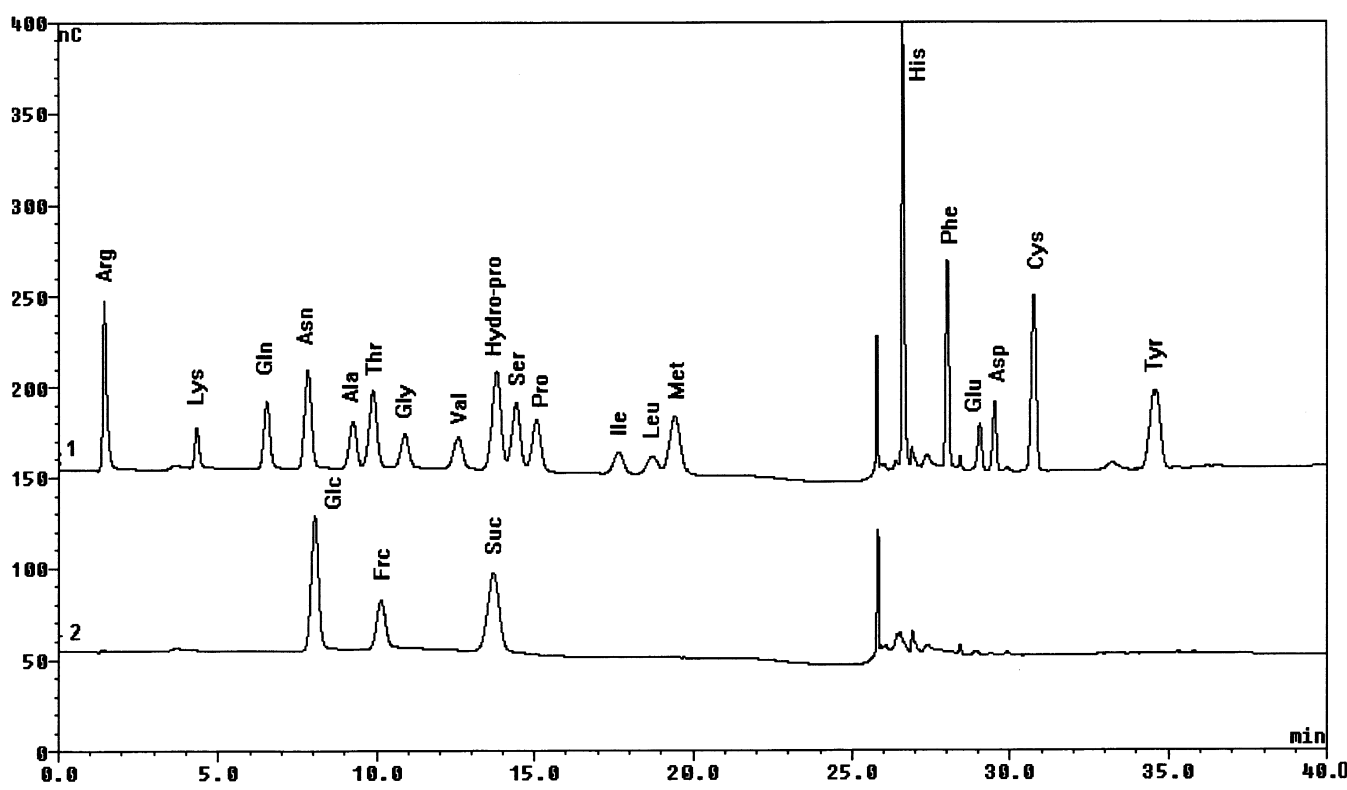

Fig. 1. Chromatograms of amino acid and sugar standards (5 $\mu M$ level). Trace 1: 20 amino acids. Trace 2: three sugars. Conditions: column, AminoPac PA10 ( $250 \times 2$ mm I.D.); eluent, see Table 1; detection waveform, see Table 2.

volume $(100 \mathrm{ml})$. Three acids $\left(\mathrm{HCl}, \mathrm{CH}_{3} \mathrm{COOH}\right.$ and $\mathrm{HCOOH}$ ) were utilized to adjust the $\mathrm{pH}$ of the water to 3.0. Chloride ion is potentially harmful to the $\mathrm{Au}$ electrode due to its corrosion behavior. Acetate ion disturbs the separation of isoleucine, leucine and methionine by changing their retention times and peak shapes. Formic acid has little influence on the analysis of amino acids using this method and is a suitable acid for adjusting the $\mathrm{pH}$ of water.

\subsection{Amino acid removal}

Both sodium hydroxide and ammonia were used to extract amino acids from the cation-exchange resin. Sodium hydroxide is undesirable for transferring amino acids from the cation exchanger due to contamination from atmospheric $\mathrm{CO}_{2}$ in the off-line mode. Ammonia is suitable as an extraction solution to elute amino acids from the cation-exchange resin. Moreover, ammonia is volatile and is readily eliminated. No adsorptive losses or destruction were observed after the evaporation step. The amino acids $(0.25 \mathrm{ml}, 50 \mu M$ level $)$ retained on the cationexchange resin $(1 \mathrm{ml})$ were completely removed by $10 \mathrm{ml}$ of $1 \mathrm{M}$ ammonia at a flow-rate of $0.5 \mathrm{ml} / \mathrm{min}$.
In addition, the effects of the concentration of ammonia solution and the amount of resin used on the losses of amino acids were examined. Only the losses of cystine are increased in the presence of increasing concentrations of ammonia $(1 \rightarrow 8 M)$ or the amount of resin used $(1 \rightarrow 4 \mathrm{ml})$, while the recoveries of the other amino acids are relatively stable.

\subsection{Evaluation of the purification method}

The reproducibility of the new method was determined by adding amino acid standards $(50 \mu M$ level) to a cation-exchange column for sample purification $(n=7, \operatorname{RSD}<5.2 \%)$. The calibration plots were tested within $0.1-20 \mu M$ and the average correlation coefficient was 0.99 . Detection limits at a signal-to-noise ratio $(S / N)$ of $>3$ for each amino acid were lower than 4.9 pmol (injection volume $25 \mu \mathrm{l})$. A recovery test was performed by spiking $0.25 \mathrm{ml}$ of amino acid standards $(50 \mu M$ level $)$ with $0.25 \mathrm{ml}$ of ratafia into the cation-exchange column and repeating each spiking experiment three times. The recovery results are shown in Table 3 . The high recoveries with low standard deviations demonstrate 
Table 3

Result of recovery and sample analysis

\begin{tabular}{lcc}
\hline Amino acid & $\begin{array}{c}\text { Recovery }(\%) \\
(\text { average } \pm \text { SD }, n=3)\end{array}$ & $\begin{array}{c}\text { Content (mg/1) in ratafia } \\
\text { (average } \pm \text { SD, } n=3)\end{array}$ \\
\hline Arginine & $98.2 \pm 2.5$ & $16.2 \pm 1.0$ \\
Lysine & $94.8 \pm 3.1$ & $3.4 \pm 0.3$ \\
Glutamine & $95.9 \pm 1.8$ & $<$ LOD \\
Asparagine & $102.2 \pm 3,0$ & $15.8 \pm 0.8$ \\
Alanine & $101.8 \pm 2.4$ & $5.5 \pm 0.2$ \\
Throenine & $104.7 \pm 2.7$ & $2.8 \pm 0.2$ \\
Glycine & $98.6 \pm 2.8$ & $4.4 \pm 0.3$ \\
Valine & $100.1 \pm 1.6$ & $1.3 \pm 0.1$ \\
Hydroxyproline & $97.8 \pm 4.0$ & $0.8 \pm 0.1$ \\
Serine & $108.1 \pm 3.0$ & $1.4 \pm 0.1$ \\
Proline & $98.6 \pm 1.5$ & $10.4 \pm 0.5$ \\
Isoleucine & $97.4 \pm 1.3$ & $<\mathrm{LOD}$ \\
Leucine & $96.8 \pm 2.0$ & $3.2 \pm 0.3$ \\
Methionine & $92.5 \pm 3.9$ & $0.8 \pm 0.05$ \\
Histidine & $104.8 \pm 4.2$ & $1.1 \pm 0.1$ \\
Phenylalanine & $96.8 \pm 2.4$ & $2.6 \pm 0.2$ \\
Glutamic acid & $92.8 \pm 3.8$ & $4.1 \pm 0.2$ \\
Aspartic acid & $96.0 \pm 3.5$ & $5.3 \pm 0.3$ \\
Cystine & $80.0 \pm 2.7$ & $0.2 \pm 0.02$ \\
Tyrosine & $93.6 \pm 3.0$ & $1.4 \pm 0.1$ \\
\hline
\end{tabular}

LOD, limit of detection.

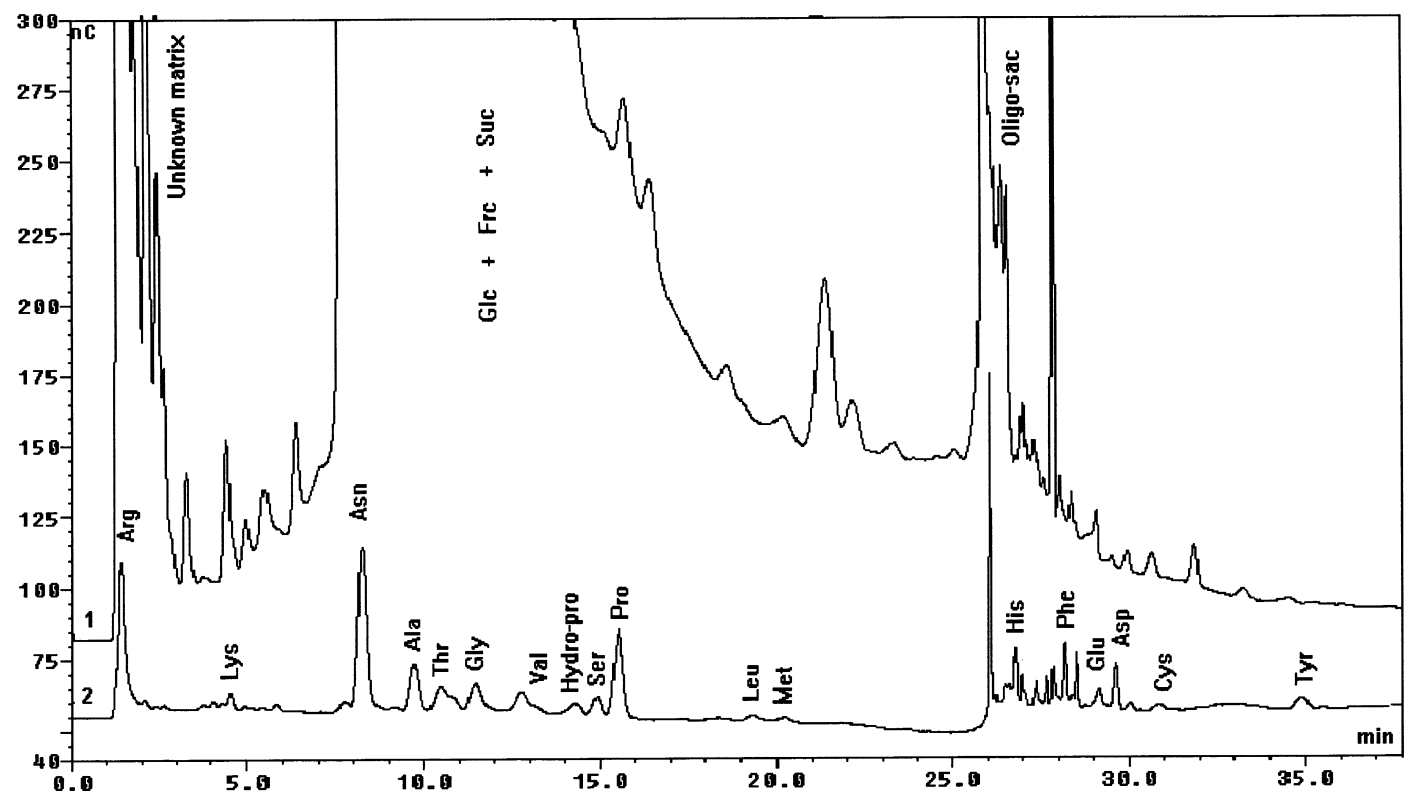

Fig. 2. Chromatograms of amino acids in ratafia. Trace 1: direct injection of ratafia diluted 100-fold. Trace 2: sample diluted 20-fold after off-line removal of carbohydrates. Conditions: see Fig. 1. 
the suitability of the method for amino acid analysis of samples with a high content of carbohydrates.

\subsection{Sample analysis}

The effectiveness of the method was tested in the analysis of ratafia samples. In Fig. 2, the chromatogram obtained without off-line sample preparation (Trace 1) reveals several major interfering peaks belonging to glucose, fructose, sucrose and oligosaccharides. The arginine peak is obscured by an unknown matrix component. In contrast, the chromatogram obtained with off-line sample preparation (Trace 2) demonstrates the complete removal of interfering carbohydrates. The matrix components masking the arginine peak are also eliminated. The content of amino acids in the sample is shown in Table 3.

\section{Conclusion}

The complete removal of large amounts of carbohydrates from a sample is possible using a strong cation exchanger with sulfonic groups. This efficient purification prior to chromatography not only prevents fouling of the column and working electrode and the potential overestimation of individual amino acids due to co-eluting compounds, but also provides a simple, flexible and economical method for amino acids analysis of samples with a high content of carbohydrates by the AEC-IPAD method.

\section{References}

[1] A.P. Clarke, P. Jandik, R.D. Rocklin, Anal. Chem. 71 (1999) 2774.

[2] Determination of the amino acid content of peptides by AAA-direct, Technical Note 50, Dionex Corporation, Sunnyvale, CA, 2000.

[3] P. Jandik, A.P. Clarke, N. Avdalovic, D.C. Andersen, J. Cacia, J. Chromatogr. B 732 (1999) 193.

[4] H. Yu, Y. Ding, S. Mou, J. Chromatogr. A 966 (2002) 89.

[5] Y. Ding, H. Yu, S. Mou, J. Chromatogr. A 982 (2002) 237.

[6] P. Jandik, J. Cheng, D. Jensen, S. Manz, N. Avdalovic, J. Chromatogr. B 758 (2001) 189.

[7] G. Bonn, J. Chromatogr. 322 (1985) 411. 